\title{
Comportamiento humano y prevención de dengue: Estudio en barrios endémicos de Barranquilla, Bucaramanga y Armenia (Colombia)
}

\section{Human behavior and prevention of dengue: Study in endemic neighborhoods of Barranquilla, Bucaramanga and Armenia (Colombia)}

Giannina Torres Pérez

Comunicadora Social y Periodista de la Universidad del Norte (Colombia) gianninatorres@gmail.com

Daniel Ernesto Aguilar Rodriguez Doctor en Sociología. Docente de la Facultad de Comunicación Social-Periodismo, Universidad Externado de Colombia. daniel.aguilar@uexternado.edu.co

Artículo de investigación

Fecha de recepción: Septiembre 20 de 2015 • Fecha de aprobación: Diciembre 20 de 2015

\section{ResUmeN}

El presente artículo expone resultados obtenidos en la investigación sobre los predictores del comportamiento que motivan el almacenamiento de agua en barrios endémicos de las ciudades de Barranquilla, Bucaramanga y Armenia (Colombia). A través de un monitoreo de almacenamiento, suministro de agua y tipo y mantenimiento de recipientes en casas centinela, así como de la implementación de un cuestionario sobre la adaptación de hogares al suministro de agua, se determinaron los factores sociales y ambientales que intervienen en el comportamiento humano, especialmente, en lo relacionado con el almacenamiento de agua. Dentro de las principales motivaciones para dicho almacenamiento se encontraron las interrupciones periódicas del suministro de agua y la necesidad de reducción de costos 
del servicio. A partir de estos hallazgos se evidencia la pertinencia de desarrollar estrategias multisectoriales, que incluyan una fase de sensibilización sobre la necesidad de generar prácticas apropiadas y seguras de almacenamiento, a fin de reducir el riesgo de producción del vector del dengue al interior de los hogares.

Palabras clave: dengue, comportamiento, clima, almacenamiento de agua.

\begin{abstract}
The present article exposes the results obtained in the research on the predictors of behavior that motivates water storage in endemic neighborhoods of Barranquilla, Bucaramanga and Armenia, in Colombia. Through monitoring the storage, water supply and type of maintenance of recipients in sentinel houses, and a questionnaire on household adaptation to water supply, it was determined the social and environmental factors involved in to human behavior, particularly related to water storage. Amongst the main motivations for such storage it was found the periodic interruptions of water supply and the need for cost reduction in the public service. Through the findings was evidenced the pertinence of developing multisector strategies, including a phase of sensitization about the need for generating appropriate and safe practices of storage, in order to reduce the production of the dengue vector within the households.
\end{abstract}

Keywords: Dengue, human behavior, climate, water storage. 
Giannina Torres Pérez, Daniel Ernesto Aguilar Rodríguez Comportamiento humano y prevención de dengue: Estudio en barrios endémicos de Barranquilla, Bucaramanga y Armenia...

\section{INTRODUCCIÓN}

El dengue aparece en el continente americano hace más de 200 años y desde 1980 se ha intensificado en número de casos, llegando a presentarse cerca de 500 millones de infectados por año (San Martín y Brathwaite, 2007; Gómez, 2015; OMS, 2015). El crecimiento demográfico constituye uno los factores que inciden en la multiplicación de la enfermedad, así como en sus resultados, entendiendo la creciente urbanización y el transporte moderno (Gubler, 2002). Las variables socio-estructurales y ambientales constituyen macro factores, en donde los profundos y desestabilizadores cambios climáticos que ocurren hoy traen consigo alteraciones de los ecosistemas (Mosquera, Obregón, Lloyd, Orozco y Peña, 2006; Parks, et al., 2004).

La infección de dengue en contextos urbanos, en donde además de los factores climáticos de temperatura y humedad (Alvis, Rodríguez y Mattal, 2015) es exacerbada por los movimientos de población incrementados por el transporte aéreo y acuático, así como por el aumento poblacional sin precedentes, marcado a su vez por procesos de desplazamiento, así como también por una urbanización desenfrenada y desestructurada que afecta severamente a las poblaciones más pobres. Los aspectos de carácter institucional también conforman macro factores, dado que los fracasos en los esquemas de erradicación y control del vector y el rápido deterioro de la infraestructura de salud pública facilita el aumento de las poblaciones de A. aegypti (Gubler, 2002; Kay y Sinh Nam, 2005). Por otra parte, los micro factores dependen de las características biológicas del virus, el vector y la persona afectada (Farrar et al., 2007), así como aspectos relacionados con el comportamiento humano, el cual está dado por una relación interdependiente entre el individuo y su entorno socio-cultural y físico.

Colombia es el país con la mayor tasa de incidencia de fiebre dengue hemorrágica en Suramérica, con un reporte anual aproximado de 40.000 casos de fiebre dengue (Padilla, Rojas y Sáenz-Gómez, 2012). Cerca del 75 \% de la población vive en medio de la cordillera de los Andes y prácticamente todas las ciudades con temperatura media entre $22 .^{\circ}$ y $30 .^{\circ}$ presentan múltiples serotipos de dengue por el contacto al ser humano - $A$. aegypti. En la actualidad el tema climático complejiza el control del mosquito, ya que estudios realizados en Australia (Queensland Government, 2016) han señalado que el riesgo en la expansión del $A$. aegypti está asociado a las acciones 
de la población humana sobre el manejo de recipientes de almacenamiento de agua, en respuesta a sus percepciones sobre el clima, determinando así un riesgo emergente de transmisión del dengue.

En el 2006 el Global Environment Facility (Fondo Mundial para el Medio Ambiente), a través del Banco Mundial, destinó fondos por cinco años a Colombia para el Integrated National Adaptation Pilot (INAP), cuyo componente de salud, con sede en el Instituto Nacional de Salud (INS), incluye el desarrollo de estrategias de adaptación al cambio climático para el dengue y la malaria.

En dos años se han obtenido avances en la predicción de las áreas de persistencia y expansión del dengue, así como en la comprensión de los efectos climáticos sobre la producción del $A$. aegypti y la identificación de intervenciones específicas sobre el comportamiento humano, como lo evidencian Padmanabha, Soto, Mosquera, Lord y Lounibos (2010). Sin embargo, mantener el control del dengue requiere de una estrategia que permita aplicar estos hallazgos en los complejos contextos comunitarios e institucionales. Más aún, se ha identificado la necesidad de entender las percepciones de la población sobre la variación climática, ya que tanto los datos de estudios preliminares, como los resultados obtenidos por Beebe, Cooper, Mottram y Sweeney (2009), han establecido el tipo de acciones sobre recipientes utilizados en respuesta a percepciones climáticas, que pueden tener mayores efectos sobre el aumento de $A$. aegypti, que los mismos efectos biológicos del aumento de temperatura. Por lo tanto, la comprensión de las percepciones sobre el cambio climático permite modelar los efectos directos e indirectos de este sobre el riesgo y control del dengue.

Además de las percepciones sobre el clima, existen otros tipos de predictores que facilitan la comprensión del comportamiento de almacenamiento de agua en los hogares. El modelo ecológico propuesto por Bronfenbrenner (1994) y revisado por Sallis, Owen y Fisher (2008) permite comprender dicho comportamiento en relación al entorno físico y sociocultural a nivel individual, interpersonal, organizacional y comunitario. Asimismo, existen estudios en torno al almacenamiento y conservación del agua, los cuales se han clasificado en actitudes, creencias, hábitos o rutinas, capacidades personales y factores del contexto (Russell y Fielding, 2010). 
Giannina Torres Pérez, Daniel Ernesto Aguilar Rodríguez Comportamiento humano y prevención de dengue: Estudio en barrios endémicos de Barranquilla, Bucaramanga y Armenia...

La comprensión integral de las motivaciones de almacenamiento de agua puede ayudar a focalizar estrategias con mayor nivel de efectividad, no tanto en la reducción de recipientes sino en la tenencia responsable de los mismos.

\section{MÉTODOS Y MATERIALES}

La investigación, de tipo mixto, se fundamentó en la observación, el monitoreo de comportamientos y la recopilación de datos sobre la adaptación de los hogares al suministro de agua, por medio de diarios de campo llevados por estudiantes de las instituciones educativas participantes en el proyecto. Esta se realizó en dos barrios endémicos de cada una de las ciudades intervenidas: Ciudadela 20 de julio y Chiquinquirá en Barranquilla ( $5 \mathrm{msnm}$ ), Campohermoso en Bucaramanga y La Cumbre, que pertenece a la municipalidad de Floridablanca $(950 \mathrm{msnm})$, y Las Colinas y La Fachada en Armenia (1550msnm). La selección se determinó a partir de los datos oficiales de las autoridades sanitarias, distrital en el caso de Barranquilla, y municipal en los casos de Armenia y Bucaramanga, entre 2004 y 2006.

Con base en categorías identificadas en investigaciones previas, como la de Padmanabha et al. (2010) o Padilla, Rojas y Sáenz (2012), que permiten determinar los comportamientos que afectan el aumento del $A$. aegypti en los hogares, se tomaron como referencias de tipo extrínseco a estos, tales como arquitectura de la vivienda (diseño de zona de lavado y recipientes de almacenamiento permanente de agua), las interacciones con el clima, como las prácticas, que difieren entre los periodos/estaciones seca y lluviosa, la demografía (edad, sexo), tamaño de la familia, la migración y los antecedentes culturales y la propiedad de la casa. Dentro de las categorías intrínsecas que, en interacción con las categorías externas, permitieron la comprensión del comportamiento específico de almacenamiento de agua, donde se encontraban las creencias, actitudes, rituales familiares, necesidades percibidas, motivaciones y costumbres (Kelly, 2001).

A fin de traducir las interacciones anteriores se diseñó un primer mapa conceptual (figura 1) que permitió establecer los factores y preguntas que se pretendían responder, como: ¿Qué factores condicionan los comportamientos de almacenamiento y 
Campos en Ciencias Sociales

El desarrollo y su complejidad desde las perspectivas multidisciplinar y transdisciplinar

vaciado de recipientes de agua?, y ¿cuáles son las percepciones de los sujetos respecto al cambio climático, que condicionan los comportamientos de almacenamiento y vaciado de recipientes de agua?

Figura 1. Mapa de categorías para el diseño del instrumento de monitoreo de comportamientos de almacenamiento de agua en casas centinela de Barranquilla, Bucaramanga y Armenia.

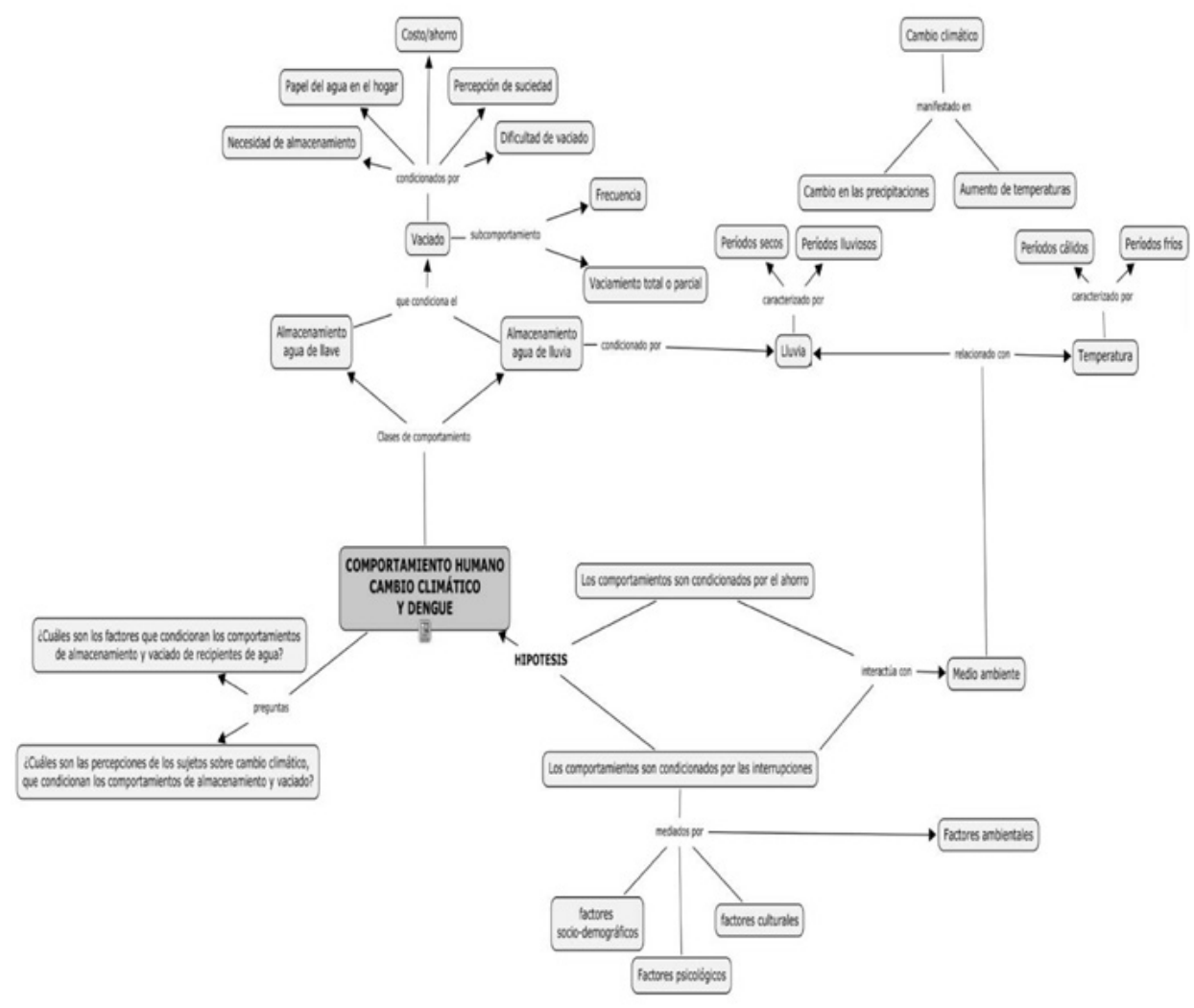

Fuente: elaboración propia. 
Como resultado de este mapa, se diseñó el primer instrumento de monitoreo de comportamientos de almacenamiento de agua en casas centinela que permitió establecer las relaciones hogar-recipiente. Dicho instrumento contaba con dos partes: 1) caracterización del hogar y los tipos y cantidad de recipientes de almacenamiento de agua en uso; 2) recopilación de datos sobre almacenamiento de agua, interrupción del servicio de agua y mantenimiento de recipientes.

Por otra parte, se implementó un segundo instrumento de observación estructurada. El formato de observación, aplicado por 40 estudiantes de bachillerato de una institución educativa oficial perteneciente a cada barrio de cada una de las tres ciudades, contempló dos componentes: el primero de ellos, un trabajo de campo individual, correspondiente al monitoreo de comportamientos y vigilancia climática; en segunda instancia, sesiones de trabajo en grupo, desarrolladas en encuentros bimensuales donde se expusieron temas relacionados con el clima, cambio climático, manejo del agua y dengue a través de talleres con los estudiantes participantes del proceso. Durante el proceso se implementaron estrategias participativas (Sánchez et al., 2008), como el uso de TIC como herramientas de comunicación y socialización, tales como una página en Facebook y un blog.

Un tercer instrumento aplicado fue una encuesta de adaptación de hogares al suministro de agua. Para su elaboración se realizó previamente un segundo mapa conceptual (figura 2) que permitió organizar el instrumento de 35 preguntas, organizadas en ocho secciones, de acuerdo a los factores descritos:

- Información sociodemográfica

- Percepciones y motivaciones para el almacenamiento de agua

- Percepciones sobre el almacenamiento y usos del agua de lluvia

- Conservación del agua

- Usos específicos del agua almacenada

- Costo

- Comportamiento en relación a la incertidumbre del servicio de agua

- Motivaciones para el no almacenamiento 
Esta encuesta se aplicó en los seis barrios que previamente fueron identificados por las secretarías de salud como endémicos, y en donde se seleccionó una muestra aleatoria simple, de 4082 casas encuestadas, de las cuales 2250 fueron encuestadas en Barranquilla: 950 correspondientes al barrio Ciudadela 20 de julio y 1300 al barrio Chiquinquirá. En Bucaramanga se aplicó el instrumento en 799 casas, 350 en el barrio Campohermoso y 449 en el barrio La Cumbre; y en Armenia en 1033 casas: 507 en el barrio Las Colinas y 526 en el barrio La Fachada. Se intervinieron las manzanas de los estudios anteriores, de acuerdo a los parches establecidos previamente, con el fin de contrastar los datos con los resultados de la investigación realizada en ańos anteriores por el proyecto INAP. 
Figura 2. Mapa de categorías para el diseño del instrumento de encuestas de adaptación de hogares al suministro de agua.

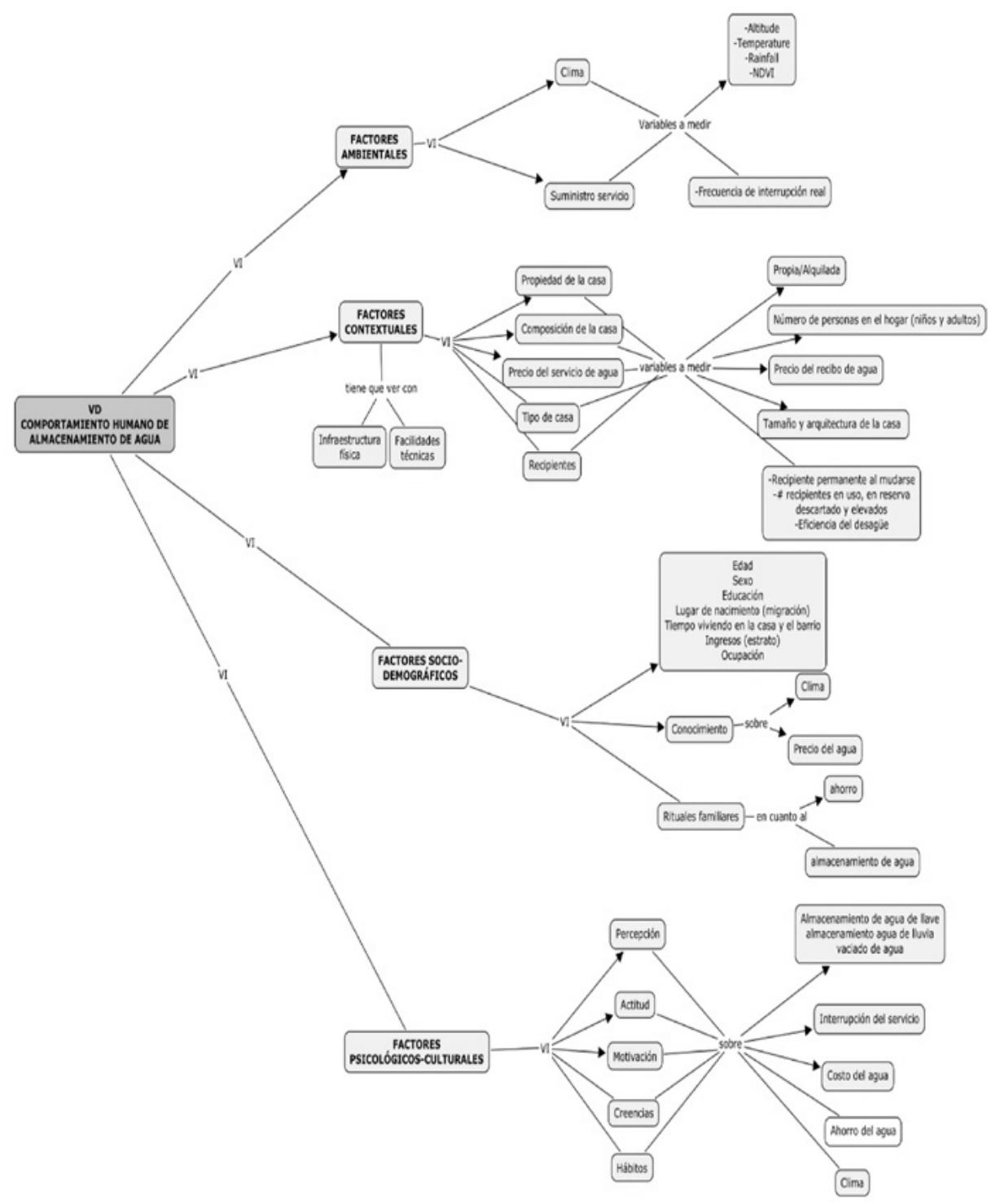

Fuente: elaboración propia. 


\section{Resultados}

\section{Caracterización de la población en estudio}

Los barrios intervenidos en el estudio pertenecen a los estratos uno, dos y tres de las ciudades intervenidas, en donde las mujeres son quienes generalmente realizan el almacenamiento de agua en sus hogares. En general, las casas son de carácter propio y el mayor porcentaje de locales visitados fueron viviendas de un bańo con inodoro útil. Las casas que pertenecían a la muestra generalmente no tenían recipiente de almacenamiento permanente.

\section{Percepciones y motivaciones para el almacenamiento de agua}

El $41 \%$ indicó que almacena agua permanentemente o cuando le avisan que habrá una interrupción en el servicio. Los recipientes de almacenamiento para uso cotidiano y reserva son los más usados. El $82 \%$ de los participantes percibe el almacenamiento de agua como un comportamiento importante para la vida. Las interrupciones del servicio, así como la necesidad de ahorro en el consumo de agua, constituyen las razones principales para el inicio de almacenamiento de agua en los hogares.

\section{Percepciones sobre el almacenamiento y usos del agua de lluvia}

De las casas en donde se llevaron a cabo las encuestas, el $95 \%$ manifestó que no almacena agua de lluvia. Las personas que sí almacenan agua de lluvia califican dicho comportamiento como muy importante para la realización de actividades como descargar el baño, regar las plantas, lavar el piso, así como agua de reserva en caso de interrupción. Por otra parte, se considera que el agua lluvia almacenada no cuenta con la calidad y potabilidad necesarias para el consumo humano.

\section{Comportamiento en relación a la incertidumbre del servicio de agua}

Las interrupciones del servicio de agua en los barrios intervenidos son, por lo general, advertidas a los habitantes, lo que permite el almacenamiento preventivo. Tales interrupciones pueden durar entre una y tres horas $\mathrm{y}$, en algunos casos, por un 
día completo, debido a reparaciones o mantenimiento en el sistema de acueducto. Cuando el servicio de agua es interrumpido, se recurre al agua almacenada para realizar actividades como bañarse, descargar el baño, lavar los platos; mientras que las actividades de lavado de la ropa, lavado del piso y regar las plantas no se realizan durante el periodo de la interrupción.

\section{Comportamiento en relación a la conservación del agua y el clima}

En cuanto a la percepción sobre al ahorro usando el agua almacenada en relación al uso del agua del grifo, en los hogares manifiestan que en actividades como bañarse, descargar el baño, lavar la ropa, regar las plantas y lavar el piso se ahorra más utilizando el agua almacenada, lo cual refuerza el comportamiento al percibirse una disminución efectiva en los costos. El $41 \%$ de los encuestados percibe el costo como alto, debido a que la mayor parte de las personas no permanecen en la residencia a lo largo del día, o porque llevan a cabo comportamientos de ahorro o de almacenamiento de agua.

Dentro de las actividades domésticas, el lavado de la ropa, bañarse, cocinar, lavar el baño y la época de vacaciones en la que llegan visitantes a las casas son consideradas como actividades que incrementan el valor del servicio de agua. En relación a la época del año en que consideran que consumen más agua, la mayoría indica que, tanto en verano como en invierno, seguido de quienes perciben que en la época de verano el consumo aumenta, ya que los habitantes de la casa se bañan más y se lava la ropa con más frecuencia.

\section{Usos específicos del agua almacenada}

En su mayoría, los hogares encuestados usan el agua almacenada de manera permanente para realizar actividades domésticas como descargar el baño o bañarse, siempre y cuando no sea con aguas lluvias. Frente al tema de facilidad de uso del agua almacenada, se percibe que en actividades como descargar el baño, lavar la ropa, lavar los platos y lavar el piso resulta más fácil, mientras que en actividades como regar las plantas se hace más difícil usando el agua almacenada. 


\section{Motivaciones para el no almacenamiento}

Dentro de las razones para el no almacenamiento de agua, se encontró que no se considera importante o necesario contar con recipientes donde almacenar el líquido. Curiosamente, la prevención de enfermedades que se generan a raíz del almacenamiento, tales como el dengue, así como la falta de tiempo para realizar el mantenimiento de los recipientes donde se almacena el agua, constituyen precisamente una de las razones más aducidas para el no almacenamiento del líquido. En cuanto a la disponibilidad para adquirir un recipiente, la mayoría manifiesta no estar dispuesto a pagar por tener un recipiente, lo cual indica que la actitud hacia el no almacenamiento es muy probable que no se modifique.

\section{Discusión}

En torno a la prevención del dengue, se ha reconocido que los comportamientos humanos influyen directamente en la producción del vector, la proliferación de la enfermedad y la generación de epidemias (Gómez y Ramsey, 2009). En este sentido, es fundamental el análisis de dichos comportamientos de almacenamiento de agua en relación a la ecología humana y del mosquito, con el fin de generar estrategias de intervención comunitaria para el control del dengue mucho más efectivas (Elder y Lloyd, 2007; Farrar et al., 2007).

De igual manera, resulta determinante tomar en cuenta los conceptos propuestos por la sicología y la sicología ambiental (Russell y Fielding, 2010) sobre los determinantes de los comportamientos de conservación del agua, dado que se encontró que las causas del comportamiento de almacenamiento de agua propuestas por Stern (2000) están directamente relacionadas con predictores como la actitud, lo que permite evaluar el comportamiento como positivo (en el caso de quienes manifestaron almacenar agua) y como negativo (para quienes no almacenan); las creencias en torno al consumo del agua y al clima como factor influyente en el comportamiento y el nivel de consumo, al percibir que en épocas como el verano el consumo era mayor porque algunas actividades domésticas eran más frecuentes; los hábitos o rutinas que se producen por una razón inicial y que refuerzan el comportamiento a lo largo del tiempo, dicha 
razón generalmente asociada con el tema de las interrupciones del servicio que creó una necesidad inicial que se convirtió en un comportamiento habitual, aun cuando la razón inicial desapareciera; las capacidades personales que tienen que ver con aspectos sociodemográficos como la edad, el sexo, el nivel educativo, los ingresos, la ocupación y el lugar de nacimiento, entre otros. Asimismo, otro de los determinantes son los factores contextuales que tienen que ver con la infraestructura física, como las facilidades técnicas, las cuales terminan por influir en la existencia o no de un hábito de almacenamiento de agua.

El seguimiento dado a las casas en donde se llevó a cabo el monitoreo, permitió identificar que las intervenciones en el tema en los barrios estudiados posibilitaran la reducción del comportamiento de almacenamiento de agua en algunos hogares. Sin embargo, las razones para el almacenamiento en los hogares que persisten en el comportamiento están muy arraigadas, lo cual reduce la posibilidad de eliminar dicho comportamiento. Ante este panorama, las recomendaciones apuntan hacia el refuerzo de estrategias de prevención que trabajen en el uso adecuado de los recipientes, en los temas de mantenimiento, limpieza y tapado.

Estudios posteriores deben profundizar en los determinantes del comportamiento humano de almacenamiento de agua y generar estrategias piloto de intervención comunitaria multisectorial a partir de dichos hallazgos. Una vez sean diseñadas y validadas estas estrategias, deben hacer parte de programas integrales de prevención y control del dengue basados en un enfoque ecosistémico, que se basa en la voluntad política de los gobiernos, la coordinación intersectorial, la participación activa de la comunidad y el fortalecimiento de la legislación nacional. 


\section{REFERENCIAS}

Alvis, N., Rodríguez, H., \& Mattar, S. (2015). Dengue in an area of the Colombian Caribbean. Colombia Médica, 46(1). Cali: Universidad del Valle. Recuperado de http:// colombiamedica.univalle.edu.co/index.php/comedica/article/view/1487

Beebe, N. W., Cooper, R. D., Mottram, P. \& Sweeney, A. W. (2009). Australia's dengue risk driven by human adaptation to climate change. PLoS Neglected Tropical Diseases, 3(429).

Bronfenbrenner, U. (1994). Ecological models of human development. International Encyclopedia of Education, 3(2). Oxford: Elsevier.

Elder, J. \& Lloyd, L. S. (2007). Achieving behaviour change for dengue control: Methods, scaling-up, and sustainability. scientific working group, report on dengue, 1-5 october 2006. World health organization on behalf of the special programme for research and training in tropical diseases. Geneva, Switzerland. Retrieved from http://www.who.int/tdr/ publications/publications/swg_dengue_2.htm

Farrar, J., Focks, D., Gubler, D., Barrera, R., Guzmán, M. G., Simmons, C., Kalayanarooj, S., Lum, L., McCall, P. J., Lloyd, L., Horstick, O., Dayal-Drager, R., Nathan, M. B. $\&$ Kroeger, A. (2007). Towards a global dengue research agenda. Tropical Medicine \& International Health, 12, (pp. 695-699).

Gómez, E. (2015). Informe del evento dengue, Colombia, hasta el periodo epidemiológico XII. Colombia: Instituto Nacional de Salud.

Gómez, H., \& Ramsey, J. (2009). Dengue in the Americas: Challenges for prevention and control. Cad. Saúde pública, 25(1).

Gubler, D. J. (2002). Epidemic dengue/dengue hemorrhagic fever as a public health, social and economic problem in the 21st century. Trends in Microbiology, 10, (pp. 100-103).

Kay, B. \& Sinh, V. (2005). New strategy against dengue in Vietnam. The Lancet, 35(59), 613-617. 
Kelly, D. (2001). Why are some people bitten more than others? Trends in parasitology, 17.

Mosquera, M., Obregón, R., Lloyd, S., Orozco, M. y Peña, A. (2006). Comunicación, movilización y participación: lecciones aprendidas en la prevención y control de la fiebre dengue (fd). Investigación y Desarrollo, 14(1), 120-151.

OMS. (2015). Number of reported cases of dengue and severe dengue (sd) in the Americas, by country: Figures for 2015 (to week noted by each country). Epidemiological Week. Recuperado de http://www.paho.org/hq/index.php?option=com_topics\&view=rdmore \&cid=6290\&Itemid $=40734$

Padilla, J., Rojas, D. y Sáenz-Gómez, R. (2012). Dengue en Colombia: epidemiología de la reemergencia a la hiperendemia. Bogotá: Ministerio de Salud de Colombia.

Padmanabha, H., Soto, E., Mosquera, M., Lord, C. C. \& Lounibos, L. P. (2010). Ecological links between water storage behaviors and Aedes Aegypti production: Implications for dengue vector control in variable climates. Ecohealth Aug, 7(1), 78-90. doi: 10.1007/ s10393-010-0301-6.

Parks, W., Lloyd, L. S., Nathan, M. B., Hosein, A., Clark, G.G., Gubler, Dawkins, Z. \& Renganathan, E. (2004). International experiences in social mobilization and communication for dengue prevention and control. Dengue bulletin, 28.

Queensland Government. (2016). About dengue. Australia. Retrieved from https://www. health.qld.gov.au/clinical-practice/guidelines-procedures/diseases-infection/diseases/ mosquito-borne/dengue/virus-fever/default.asp

Russell, S. \& Fielding, K. (2010). Water demand management research: A psychological perspective. Water resources research, 46.

Sallis, J., Owen, N. \& Fisher, E. (2008). Ecological models of health behavior. In Glanz, K., Rimer, B., \& Viswanath, K. (Ed.). Health behavior and health education: Theory, research, and practice, 4.th ed., (pp. 465-482). United States: Jossey-Bass. 
Sánchez, L., Pérez, D., Alfonso, L., Castro, M., Sánchez, L. M., Van der Stuyft, P. \& Kouri, G. (2008). Estrategia de educación popular para promover la participación comunitaria del dengue en Cuba. Revista Panamericana de Salud Pública, 24(1), 61-69.

San Martín, J. y Brathwaite-Dick, O. (2007). La estrategia de gestión integrada para la prevención y el control del dengue en la región de las Américas. Revista Panamericana de Salud Pública, 21(1), 55-63.

Stern, P. C. (2000). Toward a coherent theory of environmentally significant behavior. J. Soc. Issues, 56(3), 407-424. 